\title{
THE LIMIT BEHAVIOR OF DUAL MARKOV BRANCHING PROCESSES
}

\author{
YANGRONG LI, ${ }^{* *}$ Southwest China University and Institute of Applied Physics \\ and Computational Mathematics, Beijing \\ ANTHONY G. PAKES, ${ }^{* * *}$ University of Western Australia \\ JIA LI* AND \\ ANHUI GU,* Southwest China University
}

\begin{abstract}
A dual Markov branching process (DMBP) is by definition a Siegmund's predual of some Markov branching process (MBP). Such a process does exist and is uniquely determined by the so-called dual-branching property. Its $q$-matrix $\boldsymbol{Q}$ is derived and proved to be regular and monotone. Several equivalent definitions for a DMBP are given. The criteria for transience, positive recurrence, strong ergodicity, and the Feller property are established. The invariant distributions are given by a clear formulation with a geometric limit law.
\end{abstract}

Keywords: Markov branching process; dual Markov branching process; dual-branching $q$-matrix; transience; recurrence; strong ergodicity; invariant distribution

2000 Mathematics Subject Classification: Primary 60J27

Secondary $60 \mathrm{~J} 80$

\section{Introduction}

A (one-dimensional) Markov branching process (MBP) $Y_{t}$ is a continuous-time Markov chain (CTMC) on the state space $\mathbb{Z}_{+}=\{0,1,2, \ldots\}$ whose stochastic evolution is governed by the branching property. It was proved in [5] that an MBP is equivalent to the minimal $\tilde{\boldsymbol{Q}}$-process, where $\tilde{\boldsymbol{Q}}=\left(\tilde{q}_{i j}\right)$ is the branching $q$-matrix given by

$$
\tilde{q}_{i j}= \begin{cases}i b_{j-i+1} & \text { if } j \geq i-1, \\ 0 & \text { otherwise }\end{cases}
$$

where $b_{k} \geq 0, k \neq 1$, and $\sum_{k \geq 0} b_{k} \leq 0$, and $\tilde{\boldsymbol{Q}}$ is not assumed to be conservative. For the theory of MBPs, we refer the reader to [2] and [3] while, for the general theory of CTMCs, we refer the reader to [1].

Our aim in this paper is to discuss Siegmund's predual of an MBP which is defined formally as follows.

Received 20 October 2006; revision received 2 January 2008.

* Postal address: School of Mathematics and Statistics, Southwest China University, Chongqing, 400715, People's Republic of China.

** Email address: liyr@swu.edu.cn

*** Postal address: School of Mathematics and Statistics, University of Western Australia, 35 Stirling Highway, Crawley, WA 6009, Australia. Email address: pakes@maths.uwa.edu.au 
Definition 1.1. A process $X_{t}$ with state space $\mathbb{Z}_{+}$is called a dual Markov branching process (DMBP) if there exists an MBP $Y_{t}$ such that

$$
\mathrm{P}_{i}\left(X_{t} \geq j\right)=\mathrm{P}_{j}\left(Y_{t} \leq i\right), \quad i, j \in \mathbb{Z}_{+}, t \geq 0,
$$

where $\mathrm{P}_{i}(\cdot)=\mathrm{P}\left(\cdot \mid X_{0}=i\right)$.

Such a DMBP $X_{t}$ always exists provided that an MBP $Y_{t}$ is given. Indeed, it is well known that the MBP $Y_{t}$ always has the Feller property (see [5], [10], [13], and [14]), i.e.

$$
\mathrm{P}_{i}\left(Y_{t}=j\right) \rightarrow 0 \quad \text { as } i \rightarrow \infty \text { for every } j \in \mathbb{Z}_{+}, t \geq 0,
$$

and that $Y_{t}$ is always stochastically decreasing (see [11], [12], and [18]), i.e.

$$
\mathrm{P}_{i}\left(Y_{t} \leq j\right) \text { decreases as } i \rightarrow \infty \text { for any } j \in \mathbb{Z}_{+}, t \geq 0 \text {. }
$$

Thus, the existence of the DMBP follows from the dual form of Siegmund's theorem; see [16] and [18].

We also point out that the DMBP $X_{t}$ is in fact Siegmund's dual itself if the MBP $Y_{t}$ is honest. This is the reason why we call $X_{t}$ a dual. Indeed, the honesty assumption of $Y_{t}$ guarantees that $Y_{t}$ is stochastically monotone (or increasing), i.e.

$$
\mathrm{P}_{i}\left(Y_{t} \geq j\right) \text { increases as } i \rightarrow \infty \text { for any } j \in \mathbb{Z}_{+}, t \geq 0 .
$$

Then there exists a Siegmund-dual process $Z_{t}$ such that $\mathrm{P}_{i}\left(Z_{t} \leq j\right)=\mathrm{P}_{j}\left(Y_{t} \geq i\right)$. But, $\lim _{j \rightarrow \infty} \mathrm{P}_{j}\left(Y_{t} \geq i\right)=1$ for any honest MBP $Y_{t}$. Then $Z_{t}$ is honest. It follows that $\mathrm{P}_{i}\left(Z_{t} \geq\right.$ $j+1)=\mathrm{P}_{j}\left(Y_{t} \leq i-1\right)$, which implies (1.2) by letting $X_{t}=Z_{t}-1$.

We will prove that a DMBP is honest even if the corresponding MBP is not honest. By using this honesty, in Section 2 we derive the $q$-matrix (or transition rates) $\boldsymbol{Q}$ of a DMBP. We also derive the so-called dual-branching property and show that the DMBP is just the unique $\boldsymbol{Q}$-function that is uniquely determined by the dual-branching property (see Theorem 2.1, below). This presents several equivalent definitions of the DMBP.

In Section 3 we establish the criteria of positive recurrence, null recurrence, and transience for a DMBP. The invariant distributions (or invariant measures) are given by a clear formulation. We will see that the DMBP has a geometric limit law.

A DMBP does not necessarily have the Feller property. In Section 4 we will establish a criterion for a DMBP to be a Feller process. Aided by the Feller criterion, we establish a strong ergodicity criterion for a DMBP. In Section 5 we present some applications and examples.

It is worth pointing out that the DMBP possesses some better properties than the MBP itself (for example, honesty). Further properties are expected to be studied, and it is also possible to develop the duality theory of the generalized Markov branching processes (see [6], [7], and [9]).

\section{The transition rate and the dual-branching property}

In this section we first derive the $q$-matrix (or transition rate) $Q=P^{\prime}(0)$ componentwise, where $P(t)$ is the transition function of a DMBP.

Proposition 2.1. A DMBP is always honest. Its $q$-matrix $\boldsymbol{Q}=\left(q_{i j}\right)$ takes the following form:

$$
q_{i j}= \begin{cases}(j+1) a_{i-j}-j a_{i-j+1} & \text { if } i \geq j-1 \\ 0 & \text { otherwise }\end{cases}
$$


where $a_{k}=-\sum_{j=0}^{k} b_{j}$ for $k \geq 0$ and $\left\{b_{j}\right\}$ is the sequence for the branching q-matrix $\tilde{\boldsymbol{Q}}$ defined by (1.1). We have

$$
a_{0} \leq 0, \quad a_{1} \geq a_{2} \geq \cdots \geq a_{k} \geq \cdots \geq 0, \quad a_{-1}=0,
$$

and $a_{\infty}:=\lim _{k \rightarrow \infty} a_{k}=0$ if and only if $\tilde{\boldsymbol{Q}}$ is conservative. Furthermore, $\boldsymbol{Q}$ is regular and monotone.

Proof. Let $P(t)$ and $\tilde{P}(t)$ be the transition functions of the DMBP $X_{t}$ and the MBP $Y_{t}$, respectively. Then (1.2) can be rewritten as

$$
\sum_{k=j}^{\infty} P_{i k}(t)=\sum_{k=0}^{i} \tilde{P}_{j k}(t), \quad i, j \in \mathbb{Z}_{+} .
$$

Since 0 is an absorbing state for $Y_{t}$, it follows that $\tilde{P}_{0 j}(t)=\delta_{0 j}$ for all $j \geq 0$ and $t \geq 0$, which together with (2.3) implies that $P(t)$ is honest. Using this honesty and differentiating (2.3) at $t=0^{+}$, we obtain

$$
-\sum_{k=0}^{j-1} q_{i k}=\sum_{k=0}^{i} \tilde{q}_{j k}, \quad i \geq 0, j \geq 1
$$

which implies that

$$
q_{i j}=\sum_{k=0}^{i}\left(\tilde{q}_{j k}-\tilde{q}_{j+1, k}\right), \quad i \geq 0, j \geq 0,
$$

where $\tilde{\boldsymbol{Q}}=\left(\tilde{q}_{i j}\right)$ is just the branching $q$-matrix given by (1.1). Now let

$$
a_{k}=-\sum_{j=0}^{k} b_{j}, \quad k \in \mathbb{Z}_{+} .
$$

Then it is easy to show, from (2.5) and (1.1), that $\boldsymbol{Q}$ has the form (2.1) and, thus, (2.2) holds true. Furthermore, it is easy to verify that $\boldsymbol{Q}$ satisfies

$$
\sum_{k \geq j} q_{i k} \leq \sum_{k \geq j} q_{i+1, k} \quad \text { for all } j \neq i+1,
$$

i.e. $\boldsymbol{Q}$ is monotone. Since $\boldsymbol{Q}$ is obviously a conservative, upwardly skip-free $q$-matrix and

$$
\sum_{i=1}^{\infty} \frac{1}{q_{i, i+1}}=\frac{1}{\left|a_{0}\right|} \sum_{i=1}^{\infty} \frac{1}{i}=\infty,
$$

the regularity follows from [8, Corollary 2].

Recall that an MBP is controlled uniquely by the branching property

$$
F_{i}(s, t)=\left(F_{1}(s, t)\right)^{i} \quad \text { for all }|s| \leq 1, t \geq 0, i \in \mathbb{Z}_{+},
$$

where the generating function

$$
F_{i}(s, t):=\sum_{j \geq 0} \tilde{P}_{i j}(t) s^{j} \quad \text { for all }|s| \leq 1, t \geq 0, i \in \mathbb{Z}_{+} .
$$


The branching property (2.7) is also equivalent to

$$
\tilde{P}_{i j}(t)=\sum_{j_{1}+j_{2}+\cdots+j_{i}=j} \tilde{P}_{1 j_{1}}(t) \tilde{P}_{1 j_{2}}(t) \cdots \tilde{P}_{1 j_{i}}(t) \quad \text { for all } i, j \in \mathbb{Z}_{+}, t \geq 0
$$

We expect that a DMBP is determined uniquely by a similar property: the so-called dualbranching property, which can be derived as follows.

Theorem 2.1. Let $P(t)$ be a transition function on $\mathbb{Z}_{+}$. Then the following statements are equivalent.

(i) $P(t)$ is the transition probability of a DMBP, i.e. $P(t)$ satisfies (1.2) or, equivalently, (2.3).

(ii) $P(t)$ is the unique and honest $Q$-function, where the $q$-matrix $Q$ takes the form (2.1) and (2.2).

(iii) $P(t)$ possesses the dual-branching property

$$
P_{i j}(t)=\sum_{r=0}^{i} P_{i-r, j-1}(t)\left(P_{r-1,0}(t)-P_{r, 0}(t)\right), \quad i \geq 0, j \geq 1,
$$

where $P_{-1,0}(t) \equiv 1$.

(iv) $P(t)$ possesses the dual-branching property in terms of the generating functions

$$
G_{j}(s, t)=G_{0}(s, t)\left(1-(1-s) G_{0}(s, t)\right)^{j} \quad \text { for all }|s|<1, t \geq 0, i \in \mathbb{Z}_{+},
$$

where the generating function

$$
G_{j}(s, t):=\sum_{i \geq 0} s^{i} P_{i j}(t) \quad \text { for all }|s|<1, t \geq 0, i \in \mathbb{Z}_{+} .
$$

Proof. Statement (ii) implies statement (i). Since the $q$-matrix $\boldsymbol{Q}$, defined by (2.1) and (2.2), is monotone and regular as proved by Proposition 2.1, it follows from [18, Theorem 3.1] that the unique $\boldsymbol{Q}$-function is stochastically monotone. By Siegmund's theorem, there exists another transition function $\tilde{P}(t)$ satisfying (1.2) or, equivalently, (2.3). We need to prove that $\tilde{P}(t)$ is an MBP. Indeed, (2.3) itself implies that $\tilde{P}(t)$ has the Feller property. Thus, by [15], $\tilde{P}(t)$ is the minimal $\tilde{\boldsymbol{Q}}$-function with a stable $q$-matrix $\tilde{\boldsymbol{Q}}$. By the same method as given in Proposition 2.1 we can prove that (2.4) is true. Thus, by letting $b_{k}=a_{k-1}-a_{k}$ for $k \geq 0$, we see, from (2.1) and (2.4), that $\tilde{\boldsymbol{Q}}$ takes the form (1.1) and, thus, $\tilde{P}(t)$ is an MBP as desired.

Statement ( $i$ ) implies statement (iii). Noting that the branching property (2.8) is equivalent to (see [1])

$$
\tilde{P}_{i j}(t)=\sum_{r=0}^{j} \tilde{P}_{1, j-r}(t) \tilde{P}_{i-1, r}(t), \quad i \geq 1, j \geq 0, t \geq 0,
$$


which, together with (2.3), implies that, for $i \geq 0$ and $j \geq 1$,

$$
\begin{aligned}
P_{i j}(t) & =\sum_{k=0}^{i}\left(\tilde{P}_{j k}(t)-\tilde{P}_{j+1, k}(t)\right) \\
& =\sum_{k=0}^{i} \sum_{r=0}^{k} \tilde{P}_{1 r}(t)\left(\tilde{P}_{j-1, k-r}(t)-\tilde{P}_{j, k-r}(t)\right) \\
& =\sum_{r=0}^{i} \tilde{P}_{1 r}(t) \sum_{k=r}^{i}\left(\tilde{P}_{j-1, k-r}(t)-\tilde{P}_{j, k-r}(t)\right) \\
& =\sum_{r=0}^{i} \tilde{P}_{1 r}(t) \sum_{\tau=0}^{i-r}\left(\tilde{P}_{j-1, \tau}(t)-\tilde{P}_{j, \tau}(t)\right) \\
& =\sum_{r=0}^{i} \tilde{P}_{1 r}(t) P_{i-r, j-1}(t) .
\end{aligned}
$$

But, since $P(t)$ is honest, it follows from (2.3) again that

$$
\tilde{P}_{1 r}(t)=P_{r-1,0}(t)-P_{r, 0}(t), \quad r \geq 1, \quad \tilde{P}_{10}(t)=1-P_{00}(t) .
$$

Thus, (2.9) holds true, as desired.

Statement (iii) implies statement (ii). Differentiating (2.8) with respect to $t>0$, we obtain, for $i \geq 1$ and $j \geq 1$,

$$
P_{i j}^{\prime}(t)=\sum_{r=0}^{i}\left(P_{i-r, j-1}^{\prime}(t)\left(P_{r-1,0}(t)-P_{r 0}(t)\right)+P_{i-r, j-1}(t)\left(P_{r-1,0}^{\prime}(t)-P_{r 0}^{\prime}(t)\right)\right) .
$$

Let $\boldsymbol{Q}=\left(q_{i j}\right)$ with $q_{i j}=P_{i j}^{\prime}(0)$. It follows from (2.11) that, for $i=0$ and $j=1$,

$$
q_{0}:=-q_{00}=-\lim _{t \rightarrow 0} P_{00}^{\prime}(t)=\lim _{t \rightarrow 0} \frac{P_{01}^{\prime}(t)}{2 P_{00}(t)-1}=q_{01}<\infty,
$$

which means that 0 is a stable state. Then all the $a_{k}:=q_{k 0}, k \geq 0$, are finite and satisfy (2.2). Letting $t$ tend to 0 on both sides of (2.11), we obtain, for $i \geq 1$,

$$
q_{i j}= \begin{cases}q_{i-1, j-1}+a_{i-j}-a_{i-j+1} & \text { if } i \geq j-1, \\ q_{i-1, j-1} & \text { if } i<j-1 .\end{cases}
$$

Thus, by an induction argument we see that the $q$-matrix $Q$ has the form (2.1).

Statement (iii) is equivalent to statement (iv). If (iii) holds then (2.9) implies that

$$
1=P_{-1,0}(t) \geq \cdots \geq P_{r-1,0}(t) \geq P_{r 0}(t) \geq \cdots,
$$

and, hence,

$$
\tilde{P}_{1 r}(t):=P_{r-1,0}(t)-P_{r, 0}(t) \quad \text { for } r=0,1,2, \ldots
$$

defines a possibly defective discrete law. Let

$$
F_{1}(s, t)=\sum_{r \geq 0} \tilde{P}_{1 r}(t) s^{r}
$$


Then the generating function of this identification is

$$
F_{1}(s, t)=1-(1-s) G_{0}(s, t) .
$$

The generating function form of (2.9) is $G_{j}(s, t)=G_{j-1}(s, t) F_{1}(s, t)$ for $j \geq 1$. Iterating it gives

$$
G_{j}(s, t)=G_{0}(s, t)\left(F_{1}(s, t)\right)^{j},
$$

which, together with (2.12), implies that (2.10) holds true. Conversely, assume that (iv) holds. It is easy to show that

$$
H(s, t):=1-(1-s) G_{0}(s, t)=\sum_{r=0}^{\infty} s^{r}\left(P_{r-1,0}(t)-P_{r, 0}(t)\right) .
$$

Then (2.10) can be rewritten as

$$
G_{j}(s, t)=G_{0}(s, t)(H(s, t))^{j} .
$$

Therefore, $G_{j}(s, t)=G_{j-1}(s, t) H(s, t)$, which, together with (2.14), proves (2.9) and, thus, (iii) holds true.

\section{Recurrence and invariant distributions for a DMBP}

From now on, $\boldsymbol{Q}$ always denotes the $q$-matrix of a DMBP defined by (2.1) and (2.2). We also assume that

$$
a_{0} \neq 0 \quad \text { and } \quad a_{1} \neq 0
$$

(i.e. $Q$ is irreducible), to avoid trivial cases. We will use the following generating functions:

$$
A(s)=\sum_{j \geq 0} a_{j} s^{j}, \quad B(s)=\sum_{j \geq 0} b_{j} s^{j}, \quad C(s)=\sum_{n \geq 1} c_{n} s^{n}, \quad 0 \leq s \leq 1,
$$

where $b_{j}=a_{j-1}-a_{j}$ for $j \geq 0$ is just the sequence in the branching $q$-matrix $\tilde{\boldsymbol{Q}}$, and

$$
c_{n}:=\frac{a_{n}}{\left|a_{0}\right|} \text { for } n \geq 1 \text { and, thus, } 0 \leq c_{n} \downarrow, c_{1} \neq 0 .
$$

Then $C(s)$ is a nonnegative increasing function and has radius of convergence $r \geq 1$. Although $B(s)$ plays an important role in studying the theory of MBPs, we prefer to use $C(s)$ instead of $B(s)$, which has some advantages as we will see. Their relationship is

$$
B(s)=b_{0}(1-s)(1-C(s)) \quad \text { or, equivalently, } \quad C(s)=1-\frac{B(s)}{b_{0}(1-s)} .
$$

Thus, in the case in which $a_{\infty}=0$ or, equivalently, the case in which $\tilde{\boldsymbol{Q}}$ is conservative, the key conditions $B^{\prime}(1)<0, B^{\prime}(1)=0, B^{\prime}(1)>0$, and $B^{\prime}(1)=\infty$ are equivalent to $C(1)<1$, $C(1)=1, C(1)>1$, and $C(1)=\infty$, respectively (we see that the derivative disappears). Here we remark that if $a_{\infty} \neq 0$ then the above equivalence may not be true; we can give an example such that $C(1)=\infty$ but $B^{\prime}(1)<0$.

We now start to give the positive recurrence criterion for the DMBP. Let $P(t)$ be the unique and honest $\boldsymbol{Q}$-function. By [1, Theorem 5.1.6], the limits

$$
\pi_{j}:=\lim _{t \rightarrow \infty} P_{i j}(t), \quad i, j \in \mathbb{Z}_{+},
$$


exist and are independent of $i$ for all $j \in \mathbb{Z}_{+}$, and are either all 0 or all strictly positive. Recall that $P(t)$ is positive recurrent (or ergodic) if and only if $\sum_{j} \pi_{j}=1$, which is equivalent to $\pi_{0}>0$ since, by assumption (3.1), $P(t)$ is irreducible. In this case $\pi=\left(\pi_{j}\right)$ is the unique invariant distribution, which means that

$$
\pi P(t)=\pi \quad \text { for all } t \geq 0 .
$$

Theorem 3.1. The DMBP is positive recurrent if and only if $1<C(1) \leq \infty$ or, equivalently, if $\left|a_{0}\right|<\sum_{n \geq 1} a_{n} \leq \infty$. In this case the invariant distributions are given by

$$
\pi_{j}=(1-\xi) \xi^{j}, \quad j \in \mathbb{Z}_{+},
$$

where $\xi$ is the unique root of the equation $C(s)=1, s \in[0,1]$.

Proof. Let $\tilde{\pi}_{i j}=\tilde{P}_{i j}(\infty)$ for $i, j \geq 0$, where $\tilde{P}(t)$ is the corresponding MBP. Then letting $t$ tend to $\infty$ in (2.3) yields

$$
1-\sum_{k=0}^{j-1} \pi_{k}=\sum_{k=0}^{i} \tilde{\pi}_{j k}, \quad i \geq 0, j \geq 1 .
$$

Since the left-hand side is independent of $i$, (3.6) implies that $\tilde{\pi}_{j k}=0$ for $j \geq 1$ and $k \geq 1$ and, thus,

$$
\pi_{0}=1-\tilde{\pi}_{1,0} \quad \text { and } \quad \sum_{k=0}^{j} \pi_{k}=1-\tilde{\pi}_{j+1,0} .
$$

Therefore, the DMBP is positive recurrent (i.e. $\pi_{0}>0$ ) if and only if the extinction probability (for the MBP) $\tilde{\pi}_{1,0}<1$. But, a well-known result in the theory of MBPs shows that the extinction probability $\tilde{\pi}_{1,0}=\xi$, where $\xi$ is the minimal root of the equation $B(s)=0$, $0 \leq s \leq 1$. (Although this result seems to be well known in the conservative case only, the proof in the nonconservative case is similar to the proof in the conservative case.)

It is easy to show that the above root $\xi$ is less than 1 if and only if $1<C(1) \leq \infty$ (rather than $\left.B^{\prime}(1)>0\right)$. This has proved the first assertion of Theorem 3.1. Now assume that $C(1)>1$. Then, by (3.3), the above root $\xi$ is just the unique root of $C(s)=1,0 \leq s \leq 1$, with $0<\xi<1$, and (3.7) implies that $\pi_{0}=1-\xi$. But, letting $t$ tend to $\infty$ in the dual-branching property (2.8), we obtain $\pi_{j}=\pi_{j-1}\left(1-\pi_{0}\right)=\pi_{j-1} \xi, j \geq 1$. Iterating it gives (3.5) and completes the proof.

Remark 3.1. We also present an alternative proof of Theorem 3.1 by solving the following equation:

$$
\pi \boldsymbol{Q}=\mathbf{0}, \quad \pi=\left(\pi_{j}\right) \in l_{1}^{+},
$$

where $l_{1}^{+}$is the set of the summable sequences whose components are nonnegative. Equation (3.8) can be read as

$$
\begin{gathered}
\pi_{0} a_{0}+\pi_{1} a_{1}+\pi_{2} a_{2}+\cdots=0 \\
-n \pi_{n-1} a_{0}+\sum_{k=n}^{\infty} \pi_{k}\left((n+1) a_{k-n}-n a_{k-n+1}\right)=0, \quad n \geq 1 .
\end{gathered}
$$


Summing the preceding $n$ equations, we obtain the new equations

$$
\begin{gathered}
\pi_{0}=c_{1} \pi_{1}+c_{2} \pi_{2}+\cdots, \\
\pi_{n}=c_{1} \pi_{n+1}+c_{2} \pi_{n+2}+\cdots, \quad n \geq 1,
\end{gathered}
$$

with $\pi=\left(\pi_{j}\right) \in l_{1}^{+}$, where $c_{n}=a_{n} /\left|a_{0}\right|$ is just the sequence given in (3.2). Equations (3.9) cannot be solved recursively; we must solve (3.9) by an alternative method

If $C(1) \leq 1$ then we claim that (3.9) has only the trivial solution. Indeed, summing all equations in (3.9), we find that

$$
\sum_{n=0}^{\infty} \pi_{n}=c_{1} \sum_{n=1}^{\infty} \pi_{n}+c_{2} \sum_{n=2}^{\infty} \pi_{n}+\cdots
$$

which implies that

$$
M:=\sum_{n=0}^{\infty} \pi_{n} \leq\left(\sum_{j=1}^{\infty} c_{j}\right) M-c_{1} \pi_{0}=C(1) M-c_{1} \pi_{0} .
$$

Thus, since $0<C(1) \leq 1$ and $c_{1}>0$, it follows that $M=0$ and, thus, all $\pi_{n}=0$.

If $C(1)>1$ then there exists a unique root $\xi$ with $0<\xi<1$ of the equation $C(s)=1$, $0 \leq s \leq 1$. It is easy to verify that $\pi_{n}:=\xi^{n}, n=0,1,2, \ldots$, is a solution of (3.9) and, hence, its normalization $\pi_{n}=(1-\xi) \xi^{n}$ is also a solution of (3.8), which is just the invariant distribution and proves (3.5).

We now establish a transience criterion for the DMBP. Recall that an irreducible process is transient if

$$
\int_{0}^{\infty} P_{i i}(s) \mathrm{d} s<\infty
$$

and recurrent if

$$
\int_{0}^{\infty} P_{i i}(s) \mathrm{d} s=\infty
$$

for some (and, thus, for all) $i \in \mathbb{Z}_{+}$. The process is null recurrent if it is recurrent and $\pi_{j}=: P_{i j}(\infty)=0$. By Theorem 3.1, the transience for a DMBP may occur only in the case in which $C(1) \leq 1$.

Theorem 3.2. The DMBP is transient if and only if

$$
R:=\sum_{n=0}^{\infty} \frac{R_{n}}{n+1}<\infty
$$

where $R_{n}$ is defined recursively by $R_{0}=1$ and $R_{n}=\sum_{r=0}^{n-1} c_{n-r} R_{r}, n \geq 1$, or, equivalently, if and only if the integral

$$
I:=\int_{0}^{1} \frac{\mathrm{d} s}{1-C(s)}<\infty
$$

provided that $C(1) \leq 1$. 
Proof. By a general transience criterion in [1, Theorem 5.3.4], the DMBP is transient if and only if the equations

$$
\sum_{j=0}^{\infty} q_{i j} x_{j}=0, \quad i \neq 0,
$$

have a nonconstant bounded solution $x=\left(x_{j}, j \in \mathbb{Z}_{+}\right)$. By the form (2.1) of $\boldsymbol{Q}$, (3.12) can be read as

$$
\begin{aligned}
& 2 a_{0}\left(x_{1}-x_{2}\right)=-a_{1}\left(x_{0}-x_{1}\right), \\
&(n+1) a_{0}\left(x_{n}-x_{n+1}\right)=-a_{n}\left(x_{0}-x_{1}\right)-\cdots-n a_{1}\left(x_{n-1}-x_{n}\right), \quad n \geq 2 .
\end{aligned}
$$

Letting $y_{n}:=(n+1)\left(x_{n}-x_{n+1}\right)$ and using (3.2), we obtain

$$
\begin{gathered}
y_{1}=c_{1} y_{0}, \\
y_{n}=c_{n} y_{0}+c_{n-1} y_{1}+\cdots+c_{1} y_{n-1}, \quad n \geq 2 .
\end{gathered}
$$

By an induction argument we see that $y_{n}=R_{n} y_{0}, n \geq 0$. Also, noting that

$$
x_{0}-x_{n+1}=\sum_{k=0}^{n} \frac{y_{k}}{k+1} \text {, }
$$

we see that (3.4) has a nonconstant bounded solution if and only if

$$
\sum_{n} \frac{\left|y_{n}\right|}{n+1}<\infty \quad \Longleftrightarrow \quad R:=\sum_{n} \frac{R_{n}}{n+1}<\infty,
$$

which proves the first assertion of the theorem.

Now assume that $C(1) \leq 1$, and let

$$
R(s):=\sum_{n} R_{n} s^{n}
$$

Then it is easy to show that $R(s)=1+C(s) R(s)$ and, thus, $R(s)=1 /(1-C(s))$. Since

$$
T(s):=\int_{0}^{s} R(\tau) \mathrm{d} \tau=\sum_{n=0}^{\infty} \frac{R_{n}}{n+1} s^{n+1}, \quad 0 \leq s<1,
$$

it follows that

$$
R=T(1-)=\int_{0}^{1} R(s) \mathrm{d} s=\int_{0}^{1} \frac{\mathrm{d} s}{1-C(s)},
$$

which shows that (3.11) is equivalent to (3.10). This completes the proof.

As a consequence, we have the following easy-to-check conditions.

Corollary 3.1. Let $\boldsymbol{Q}$ be the dual-branching $q$-matrix defined by (2.1), (2.2), and (3.1).

(i) If $C(1)>1$ then the $D M B P$ is positive recurrent.

(ii) If $C(1)<1$ then the DMBP is transient.

(iii) If $C(1)=1$ and $C^{\prime}(1)<\infty$ (here $\left.C^{\prime}(1)=\sum_{n \geq 1} n c_{n}\right)$ then the DMBP is null recurrent. 
(iv) If $C(1)=1$ and $C^{\prime}(1)=\infty$ then the DMBP is null recurrent or transient according to whether $R=I=\infty$ or $R=I<\infty$, respectively.

Note that, by Corollary 3.1, for the case in which $C(1)=1$ and $C^{\prime}(1)=\infty$, the result is not so clear as for cases (i)-(iii). Indeed, examples will be given in Section 5 (see Examples 5.2 and 5.3, below) to show that, for this case, both transience and null recurrence may occur.

\section{Strong ergodicity and the Feller property}

In this section we are concerned with how fast the DMBP $P_{i j}(t)$ convergences to the ergodic limit $\pi_{j}$. Recall that $P(t)$ is strongly ergodic if

$$
\sup _{i} \sum_{j}\left|P_{i j}(t)-\pi_{j}\right| \rightarrow 0 \quad \text { as } t \rightarrow \infty .
$$

For the details, we refer the reader to [1]. Aided by the Feller properties, we can establish the strong ergodicity criterion for the DMBP as follows.

Theorem 4.1. Let $\boldsymbol{Q}$ be the dual-branching q-matrix defined by (2.1), (2.2), and (3.1).

(i) If $a_{\infty}:=\lim _{k \rightarrow \infty} a_{k} \neq 0$ then the DMBP is strongly ergodic.

(ii) If $a_{\infty}=0$ then the DMBP is strongly ergodic if and only if $C(1)=\infty$ and there exists some $\varepsilon$ with $\xi<\varepsilon<1$ such that the integral

$$
I(\varepsilon):=\int_{\varepsilon}^{1} \frac{1}{(C(s)-1)(1-s)} \mathrm{d} s<\infty,
$$

where $\xi$ is the unique root of $C(s)=1,0 \leq s \leq 1$.

To give a proof of Theorem 4.1, we need to establish a criterion for the DMBP to be a Feller process, where the Feller property is defined by (1.3) and investigated in [5], [10], [12], and [18]. It is worth pointing out that the Feller property itself is also a limit behavior. In fact, it describes the asymptotic behavior at the remote state (i.e. as $i$ tends to $\infty$ ).

Lemma 4.1. Assume that $a_{\infty}=0$. Then the following statements are equivalent to each other.

(a) The DMBP $X_{t}$ is a Feller process.

(b) The corresponding MBP $Y_{t}$ is honest.

(c) Either $C(1)<\infty$ or $C(1)=\infty$ and $I(\varepsilon)=\infty$ for some (and, thus, for all) $\varepsilon$ with $\xi<\varepsilon<1$, where $\xi$ and $I(\varepsilon)$ are as given in Theorem 4.1.

Proof. Statement (a) is equivalent to statement (b). By [11, Lemma 4.4], a stochastically monotone process has the Feller property if and only if its Siegmund dual (or dualizee in the sense of [5]) is also stochastically monotone. Thus, the dual MBP $X_{t}$ has the Feller property if and only if the corresponding MBP $Y_{t}$ is stochastically monotone. But, by the assumption that $a_{\infty}=0$, the branching $q$-matrix $\tilde{\boldsymbol{Q}}$ is conservative and, thus, $\tilde{\boldsymbol{Q}}$ is monotone, i.e. $\tilde{\boldsymbol{Q}}$ satisfies (2.6). (In fact, it is easy to verify that the branching $q$-matrix $\tilde{\boldsymbol{Q}}$ is monotone if and only if $\tilde{\boldsymbol{Q}}$ is conservative.) Since the MBP $Y_{t}$ must be the minimal $\tilde{\boldsymbol{Q}}$-function and $\tilde{\boldsymbol{Q}}$ is monotone, it follows from [18, Theorem 3.1] that $Y_{t}$ is stochastically monotone if and only if $\tilde{\boldsymbol{Q}}$ is regular, which means that $Y_{t}$ is honest. 
Statement $(b)$ is equivalent to statement $(c)$. By the standard regularity criterion for a conservative MBP (see [1], [2], and [3]), the MBP $Y_{t}$ is honest if and only if either $B^{\prime}(1)<\infty$ or $B^{\prime}(1)=\infty$ and

$$
\int_{\varepsilon}^{1} \frac{1}{B(s)} \mathrm{d} s=-\infty
$$

for some $\varepsilon$ with $\xi<\varepsilon<1$, where $\xi$ is the minimal root of $B(s)=0,0 \leq s \leq 1$. By (3.3) and the assumption that $a_{\infty}=0$, this is also equivalent to either $C(1)<\infty$ or $C(1)=\infty$ and

$$
I(\varepsilon)=\int_{\varepsilon}^{1} \frac{\mathrm{d} s}{(1-s)(C(s)-1)}=\infty
$$

for some $\varepsilon$ with $\xi<\varepsilon<1$, where $\xi$ is the unique root of $C(s)=1,0 \leq s \leq 1$. Obviously, $\xi<1$ since $C(1)=\infty$.

Proof of Theorem 4.1. (i) Assume that $a_{\infty} \neq 0$. By [1, Theorem 5.2.3] we know that the DMBP $X_{t}$ is strongly ergodic if and only if the system

$$
\sum_{j \neq 0} q_{i j} x_{j} \leq-1, \quad i \neq 0,
$$

has a bounded nonnegative solution $\boldsymbol{x}=\left(x_{i}\right)$. Take

$$
\boldsymbol{x}=\left(0, \frac{1}{a_{\infty}}, \frac{1}{a_{\infty}}, \ldots, \frac{1}{a_{\infty}}, \ldots\right)^{\top},
$$

where ' $\top$ ' denotes the transpose, then it is easy to verify that, for $i \geq 1$,

$$
\sum_{j \neq 0} q_{i j} x_{j}=\sum_{j=1}^{i+1}\left((j+1) a_{i-j}-j a_{i-j+1}\right) \frac{1}{a_{\infty}}=-\frac{a_{i}}{a_{\infty}} \leq-1,
$$

which means that $\boldsymbol{x}$ is indeed a bounded solution of (4.1) and, thus, the DMBP is strongly ergodic.

(ii) Now assume that $a_{\infty}=0$. If the DMBP $X_{t}$ is strongly ergodic then, by [18, Theorem 2.1], $X_{t}$ is not a Feller process. Thus, applying Lemma 4.1 we must have $C(1)=\infty$ and $I(\varepsilon)<\infty$. Conversely, if $C(1)=\infty$ and $I(\varepsilon)<\infty$ then, by Lemma 4.1 again, the DMBP is not a Feller process. Since $C(1)=\infty>1$, it follows from Theorem 3.1 that $X_{t}$ is ergodic (i.e. positive recurrent). The DMBP $X_{t}$ is also stochastically monotone. Thus, the strong ergodicity of $X_{t}$ follows from [18, Theorem 2.2]. (This theorem proved that a monotone and ergodic process is strongly ergodic if and only if it is not a Feller process.)

Corollary 4.1. If $a_{\infty} \neq 0$ or $C(1)=\infty$ and $I(\varepsilon)=\infty$, then the DMBP is exponentially ergodic.

Remark 4.1. As a consequence of Theorem 4.1, we know that if $a_{\infty} \neq 0$ then the DMBP is not a Feller process in view of [17, Theorem 2.1]. On the other hand, we have $I(\varepsilon)<\infty$ if $a_{\infty} \neq 0$. Therefore, Lemma 4.1 is always true no matter whether $a_{\infty}=0$ or $a_{\infty} \neq 0$, and it gives a Feller criterion for the DMBP in the general case. As an aside, we also find that a nonconservative branching $q$-matrix is not zero-exit. We also remark here that a nonconservative MBP is not honest with the extinction probability $\tilde{P}_{1,0}(\infty)<1$ even though $B^{\prime}(1) \leq 0$. But, it is true that $\tilde{P}_{1,0}(\infty)<1$ if and only if $C(1)>1$ no matter whether in the conservative case or in the nonconservative case. This is an advantage of using the generating function $C(s)$ instead of $B(s)$. 
Remark 4.2. Together with all the information provided in this paper, a clear picture for the DMBP may be given as follows: a DMBP is

(i) honest, regular, and stochastically monotone always (even though the corresponding MBP is not);

(ii) transient if and only if either $C(1)<1$ or $C(1)=1, C^{\prime}(1)=\infty$, and $R<\infty$;

(iii) null recurrent if and only if either $C(1)=1$ and $C^{\prime}(1)<\infty$ or $C(1)=1, C^{\prime}(1)=\infty$, and $R=\infty$;

(iv) ergodic (or positive recurrent) if and only if $1<C(1) \leq \infty$;

(v) strongly ergodic if and only if $C(1)=\infty$ and $I(\varepsilon)<\infty$;

(vi) a Feller process if and only if either $C(1)<\infty$ or $C(1)=\infty$ and $I(\varepsilon)=\infty$.

\section{Examples and applications}

Note that the dual-branching $q$-matrix $\boldsymbol{Q}$ defined by (2.1) and (2.2) has the upwardly skipfree structure. If we consider some special cases of DMBPs then we can obtain some birth-death processes with catastrophes, which have been investigated in [4].

Example 5.1. (Uniform catastrophes.) Let $\boldsymbol{Q}=\left(q_{i j}\right)$ be a dual-branching $q$-matrix satisfying (2.1) and (2.2). If we take $a_{i} \equiv d>0$ for $i \geq 1$ and $a_{0}=-\lambda<0$ then the $q$-matrix $Q$ can be read as

$$
q_{i j}= \begin{cases}d & \text { if } i \geq j+1 \\ (j+1) \lambda-j d & \text { if } i=j \\ -j \lambda & \text { if } i=j-1 \\ 0 & \text { if } i<j-1\end{cases}
$$

The above $q$-matrix is just the uniform catastrophes $q$-matrix introduced in [4] (or see [1, p. 302]). Using our conclusions on the DMBP, we have the following result.

Proposition 5.1. Suppose that the $q$-matrix $Q$ is defined by (5.1). Then

(i) $\boldsymbol{Q}$ is regular and, thus, the minimal $\boldsymbol{Q}$-process is the unique $\boldsymbol{Q}$-process;

(ii) the unique $\boldsymbol{Q}$-process is strongly ergodic;

(iii) the unique $\boldsymbol{Q}$-process is not Feller;

(iv) the invariant distribution $\pi=\left(\pi_{j}\right)$ is given by

$$
\pi_{j}=\frac{\lambda d^{j}}{(\lambda+d)^{j+1}}, \quad j \in Z_{+} .
$$

Proof. The proof of part (i) follows from Proposition 2.1. Since $\lim _{n \rightarrow \infty} a_{n}=d>0$, the strong ergodicity follows from Theorem 4.1. The proof of part (iii) follows from Remark 4.2. We need only prove (5.2). Note that the generating function

$$
C(s)=\sum_{n=1}^{\infty} \frac{\lambda}{d} s^{n}=\frac{\lambda s}{d(1-s)} .
$$


Solving the equation $C(s)=1,0<s<1$, we obtain the unique root

$$
\xi=\frac{d}{\lambda+d}
$$

which, together with (3.5), implies that (5.2) holds true.

To close the paper, we give two examples to illustrate that both transience and null recurrence can occur in the case in which $C(1)=1$ with $C^{\prime}(1)=\infty$.

Example 5.2. We take the dual-branching $q$-matrix $\boldsymbol{Q}$ with

$$
a_{0}=-1, \quad a_{n}=c_{n}=\frac{(-1)^{n+1}}{n !} \alpha(\alpha-1) \cdots(\alpha-n+1), \quad n \geq 1,
$$

where the constant $\alpha \in(0,1)$. Then $\left\{a_{n}\right\}$ satisfies (2.2) and $0<c_{n} \downarrow 0$. The generating function is given by

$$
C(s)=1-(1-s)^{\alpha} .
$$

It is easy to verify that $C(1)=1$ and $C^{\prime}(1)=\infty$. But, the integral in (3.11) is

$$
I=\int_{0}^{1} \frac{\mathrm{d} s}{1-C(s)}=\int_{0}^{1}(1-s)^{-\alpha} \mathrm{d} s<\infty .
$$

Thus, by Theorem 3.2, the DMBP is transient.

Example 5.3. We now take

$$
a_{0}=-1, \quad a_{n}=c_{n}=\frac{1}{n(n+1)}, \quad n \geq 1 .
$$

Then $\left\{a_{n}\right\}$ satisfies (2.2). It is easy to verify that $C(1)=1$ and $C^{\prime}(1)=\infty$. We also have

$$
1-C(s)=1-\sum_{n=1}^{\infty} \frac{1}{n} s^{n}+\sum_{n=1}^{\infty} \frac{1}{n+1} s^{n}=\frac{(s-1) \log (1-s)}{s}, \quad 0<s<1 .
$$

Thus, the integrals

$$
I=\int_{0}^{1} \frac{\mathrm{d} s}{1-C(s)} \geq \frac{1}{2} \int_{1 / 2}^{1} \frac{\mathrm{d} s}{(s-1) \log (1-s)}=-\frac{1}{2} \int_{0}^{1 / 2} \frac{\mathrm{d} s}{s \log s}=\infty .
$$

By Corollary 3.1, the DMBP is null recurrent.

\section{Acknowledgements}

This work has been supported by China Postdoctoral Science Foundation (grant number 2005038326).

The authors wish to thank an anonymous referee for providing helpful suggestions. 


\section{References}

[1] Anderson, W. J. (1991). Continuous Time Markov Chains. Springer, New York.

[2] Asmussen, S. And Hering, H. (1983). Branching Processes. Birkhäuser, Boston, MA.

[3] Athreya, K. B. And Ney, P. E. (1972). Branching Processes. Springer, Berlin.

[4] Brockwell, P. J., Gani, J. And Resnick, S. I. (1982). Birth, immigration and catastrophe processes. Adv. Appl. Prob. 14, 709-731.

[5] Chen, A. Y. (2001). Applications of Feller-Reuter-Riley transition functions. J. Math. Anal. Appl. 260, $439-456$.

[6] Chen, A. Y. (2002). Ergodicity and stability of generalized Markov branching processes with resurrection. $J$. Appl. Prob. 39, 786-803.

[7] Chen, A. Y. (2002). Uniqueness and extinction properties of generalized Markov branching processes. J. Math. Anal. Appl. 274, 482-494.

[8] Chen, A. Y., Pollett, P., Zhang, H. J. and Cairns, B. (2005). Uniqueness criteria for continuous-time Markov chains with general transition structures. Adv. Appl. Prob. 37, 1056-1074.

[9] Chen, R. R. (1997). An extended class of time-continuous branching processes. J. Appl. Prob. 34, 14-23.

[10] LI, Y. R. (2003). Contraction integrated semigroups and their application to continuous-time Markov chains. Acta Math. Sinica English Ser. 19, 605-618.

[11] LI, Y. R. (2006). Dual and Feller-Reuter-Riley transition functions. J. Math. Anal. Appl. 313, 461-474.

[12] LI, Y. R. (2007). Strongly monotone transition functions and a note on strong ergodicity of monotone $q$-functions. Statist. Prob. Lett. 77, 396-400.

[13] PAKEs, A. G. (1981). The limit behaviour of a Markov chain related to the simple branching process allowing immigration. J. Math. Phys. Sci. 15, 159-171.

[14] Pakes, A. G. (1993). Explosive Markov branching processes: entrance laws and limiting behavior. Adv. Appl. Prob. 25, 737-756.

[15] Reuter, G. E. H. And Riley, P. W. (1972). The Feller property for Markov semigroups on a countable state space. J. Lond. Math. Soc. 5, 267-275.

[16] SiEgmund, D. (1976). The equivalence of absorbing and reflecting barrier problems for stochastically monotone Markov processes. Ann. Prob. 41, 914-924.

[17] Zhang, H. J. And Chen, A. Y. (1999). Stochastic comparability and dual $q$-functions. J. Math. Anal. Appl. 234, 482-499.

[18] Zhang, H. J., Chen, A. Y., Lin, X. And Hou, Z. (2001). Strong ergodicity of monotone transition functions. Statist. Prob. Lett. 55, 63-69. 